\title{
HPV vaccination in Japan: results of a 3-year follow-up survey of obstetricians and gynecologists regarding their opinions toward the vaccine
}

\section{Osaka University}

Masaaki Sawada, Yutaka Ueda, Asami Yagi, Akiko Morimoto, Ruriko Nakae, Reisa Kakubari, Hazuki Abe, Shinya Matsuzaki, Eiji Kobayashi, Tadashi Kimura

[Background]

In Japan, the cervical cancer preventative HPV vaccination rate has dramatically declined, directly as a result of repeated broadcasts of so-called adverse events and the resulting suspension of the government's recommendation. Our previous survey of obstetricians and gynecologists in Japan regarding their opinions toward HPV vaccination revealed that these key specialists were as negatively influenced by the reports of purported negative events as were the general population. Here, we report a 3-year follow-up survey of these clinicians.

[Methods]

We reused the same questionnaire format as used in our 2014 survey, but added new questions concerning opinions regarding a WHO statement and reports of a Japanese nation-wide epidemiological study related to the adverse events, released in 2015 and 2016 , respectively.

[Results]

\section{$\underline{\text { Characteristics of the responders }}$}

Previous survey (By 2014, a response rate of 46\%)

\begin{tabular}{|l|c|l|c|}
\hline \multicolumn{2}{|l|}{} & $\mathrm{n}(\%)$ & Age \\
\hline Male & $169(64)$ & Twenties & $7(3)$ \\
\hline Female & $95(36)$ & Thirties & $65(25)$ \\
\hline Working status & Forties & $52(67)$ \\
\hline Employed as a doctor & $160(61)$ & Fifties & $67(25)$ \\
\hline Owner of a clinic & $92(35)$ & Sixties & $40(15)$ \\
\hline Others & $11(4)$ & Seventies & $33(13)$ \\
\hline
\end{tabular}

Present survey (By 2017, a response rate of 46\%)

\begin{tabular}{|l|c|l|c|}
\hline \multicolumn{2}{|l|}{} & $\mathrm{n}(\%)$ & Age \\
\hline Male & $167(65)$ & Twenties & $6(2)$ \\
\hline Female & $89(35)$ & Thirties & $58(23)$ \\
\hline Working status & $148(58)$ & Fifties & $58(23)$ \\
\hline Employed as a doctor & $91(35)$ & Sixties & $51(20)$ \\
\hline Owner of a clinic as a doctor & $17(7)$ & Seventies & $32(12)$ \\
\hline Others & & & \\
\hline
\end{tabular}

Comparison of baseline opinions toward HPV vaccine between the previous and the present survey

\begin{tabular}{|c|c|c|c|}
\hline & Previous survey & Present survey & $p$ value \\
\hline Government should restart its recomendation & $\begin{array}{c}61.0 \% \\
(161 / 264)\end{array}$ & $\begin{array}{c}73.6 \% \\
(187 / 254) \\
\end{array}$ & 0.0027 \\
\hline I recommend HPV vaccine for teenagers & $\begin{array}{c}65.2 \% \\
(172 / 264)\end{array}$ & $\begin{array}{c}70.1 \% \\
(178 / 254)\end{array}$ & 0.26 \\
\hline I had my teenaged daughter inoculated & $\begin{array}{c}0 \% \\
(0 / 18)\end{array}$ & $\begin{array}{l}16.1 \% \\
(5 / 31)\end{array}$ & 0.14 \\
\hline
\end{tabular}

The rate of government should restart its recommendation was significantly higher $(\mathrm{p}=0.0027)$ than that in the previous study. Interestingly, in the present survey, $5(16.1 \%)$ of 31 doctors had their own teenaged daughters inoculated with HPV vaccine, even though the governmental recommendation was still suspended.
World Health Global Advisory Committee on Vaccine safety Organization Statement on Safety of HPV vaccines 17 December 2015

\section{Report of epidemiological study ; The Sobue Report}

The GACVS has systematically investigated safety concerns raised about HPV vaccines and has issued several reports in this regard. To date, it has not found any safety issue that would alter its recommendations for the use of the vaccine. (snip) The circumstances in Japan, where the occurrence of chronic pain and other symptoms in some vaccine recipients has led to suspension of the proactive recommendation for routine use of vaccine in the national immunization program, warrants additional comment. Review of clinical data by the national expert committee led to a conclusion that symptoms were not related to the vaccine, but it has not been possible to reach consensus to resume HPV vaccination. As a result, young women are being left vulnerable to HPV-related cancers that otherwise could be prevented. As GACVS has noted previously, policy decisions based on weak evidence, leading to lack of use of safe and effective vaccines, can result in real harm.

Survey answers regarding the WHO statement and the report of Japanese nation-wide epidemiological study (the Sobue Report)

\begin{tabular}{|l|c|c|}
\hline & Was aware & Confirming safety/effectiveness \\
\hline WHO statement & $\begin{array}{c}61.0 \% \\
(161 / 264)\end{array}$ & $\begin{array}{c}73.6 \% \\
(187 / 254)\end{array}$ \\
\hline $\begin{array}{l}\text { Report of epidemiological study } \\
\text { (the Sobue Report) }\end{array}$ & $\begin{array}{c}71.5 \% \\
(178 / 249)\end{array}$ & $\begin{array}{c}84.3 \% \\
(209 / 248)\end{array}$ \\
\hline \hline
\end{tabular}

The conclusion of a nation-wide epidemiologic study of adolescents in Japan regarding diverse symptoms, including pain and motor disability, conducted under MHLW financial support

1. There were adolescents who did not receive HPV vaccination who suffered from symptoms similar to those observed in HPV vaccinated girls.

2. This study could not evaluate the casual association of HPV vaccination on diverse symptoms observed in vaccinated girls.

Change of opinions toward HPV vaccine after reading information (the WHO statement and the Sobue Report)

\begin{tabular}{||l|c|}
\hline & $\begin{array}{c}\text { Positive change with } \\
\text { the information provided }\end{array}$ \\
\hline $\begin{array}{l}\text { Government should restart } \\
\text { recommendation }\end{array}$ & $\begin{array}{c}17.9 \% \\
(12 / 67)\end{array}$ \\
\hline $\begin{array}{l}\text { I recommend HPV vaccine for } \\
\text { teenagers }\end{array}$ & $6.6 \%$ \\
\end{tabular}

Among the responders, about $70 \%$ were aware of the WHO statement and the Sobue Report. Further, about $70 \%$ to $80 \%$ of respondents agreed with the safety and the effectiveness of the HPV vaccine. Among those, each of $17.9 \%$ and 6.6\% changed their answer to 'Yes' after reading at the WHO statement and the Sobue Report.

\section{[Conclusion]}

Our re-survey of Japan's obstetricians and gynecologists regarding their opinions about the HPV vaccine found that their opinions have changed, potentially leading to a more positive future re-engagement for HPV vaccination in Japan. 